\title{
La frontera Colombia-Perú vista a través de la Operación de Apoyo al Desarrollo Binacional*
}

| Fecha de recibido: 17 de marzo del 2021 | Fecha de aprobación: 8 de abril del 2021 |

* Este artículo de investigación científica es resultado del Proyecto de Iniciación Científica PIC-DIS-3306, financiado por la Vicerrectoría de Investigaciones de la Universidad Militar Nueva Granada durante el periodo 2020-2021, denominado "Seguridad y Desarrollo en la Frontera Colombia-Perú a través de la Operación de Apoyo al Desarrollo Binacional, en el periodo 2007-2019".

\section{González-Cuenca}

Magíster en Relaciones

y Negocios Internacionales

Universidad Militar Nueva Granada

Colombia

Grupo de investigación: Procesos de Integración

Regionalización y Estructuras Organizacionales Rol de investigador: teórico, experimental y escritura https://orcid.org/0000-0002-6563-9193

$\triangle$ david.gonzalez@unimilitar.edu.co

\section{Héctor Mauricio \\ Cano Rodríguez}

Especialidad tecnológica en Control Naval de Tráfico Marítimo

Universidad Militar Nueva Granada Colombia

Grupo de investigación: Semillero PIREO

Rol de investigador: recolección de información experimental y escritura https://orcid.org/0000-0002-8614-5985

\6903741@unimilitar.edu.co

Cómo citar este artículo: González-Cuenca, D., \& Cano Rodríguez, H. M. (2021). La frontera Colombia-Perú vista a través de la Operación de Apoyo al Desarrollo Binacional. Ciencia y Poder Aéreo, 16(1), 102-115, https://doi.org/10.18667/cienciaypoderaereo.711 


\section{La frontera Colombia-Perú
vista a través de la Operación \\ vista a través de la Operación de Apoyo al Desarrollo Binacional}

Resumen: Este artículo hace un estudio cualitativo, con enfoque analítico e inductivo, sobre las implicaciones, en materia de seguridad y desarrollo, de la implementación de la Operación de Apoyo al Desarrollo Binacional en la frontera colombo-peruana, durante el periodo 20072019. Para ello, se especificaron las acciones más representativas de la ejecución de la operación; se determinó la evolución de la calidad de vida, a partir de la conceptualización del desarrollo derivada de la operación, y, finalmente, se estableció el alcance y el cumplimento de las metas y objetivos propuestos por la Operación de Apoyo al Desarrollo Binacional Colombia-Perú. Este análisis permitió establecer, por un lado, la importancia de fortalecer operaciones como la de Apoyo al Desarrollo Binacional en esta zona de frontera y en otras de similares características, con el fin de impulsar el desarrollo territorial de la mano de los pobladores de la región, así como, por otro lado, la necesidad de vincular a más instituciones públicas, privadas y gobiernos extranjeros en la construcción de escenarios de cooperación internacional, en beneficio del desarrollo y la seguridad, en este caso particular, de la zona ribereña de los ríos Putumayo y Amazonas, territorios fronterizos entre Colombia y Perú.

Palabras clave: Colombia; Perú; cooperación internacional; desarrollo; frontera; seguridad.

Abstract: This paper presents a qualitative study, under an analytical and inductive approach, of the implications, in terms of security and development, of the Binational Development Support Operation on the Colombia-Peru border during 2007-2019. To do this, the most representative actions in the execution of said operation were specified, in addition to determining the evolution of the quality of life in this area, based on the concept of the development derived from the operation. The scope and fulfillment of the goals and objectives proposed by the Colombia-Peru Binational Development Support Operation were also addressed in this study. This analysis established, on the one hand, the importance of strengthening this kind of operations in the border area studied, and others with similar characteristics, in order to promote territorial development together with local inhabitants. On the other hand, findings point to the need of engaging more public and private institutions and foreign governments in the construction of international cooperation scenarios for the benefit of development and security, in this particular case, of the riparian zone of rivers Putumayo and Amazonas, bordering territories between Colombia and Peru.

Keywords: Colombia; Peru; international cooperation; development; border; security.

Resumo: Este artigo faz um estudo qualitativo, com enfoque analítico e indutivo, sobre as implicações, em matéria de segurança e desenvolvimento, da implementação da Operação de Apoio ao Desenvolvimento Binacional na fronteira colombo-peruana, durante o período 2007-2019. Para o efeito, foram especificadas as ações mais representativas da execução da operação; a evolução da qualidade de vida foi determinada com base na conceituação do desenvolvimento resultante da operação e, por último, foram estabelecidos o propósito e o cumprimento das metas e objetivos propostos pela Operação de Apoio ao Desenvolvimento Binacional Colômbia-Peru. Esta análise permitiu estabelecer, por um lado, a importância de fortalecer operações como a de Apoio ao Desenvolvimento Binacional nesta zona de fronteira e em outras de características semelhantes, a fim de promover o desenvolvimento territorial da mão dos habitantes da região, bem como a necessidade de associar mais instituições públicas, privados e governos estrangeiros na construção de cenários de cooperação internacional, em benefício do desenvolvimento e da segurança, neste caso específico, da zona ribeirinha dos rios Putumayo e Amazonas, territórios fronteiriços entre a Colômbia e o Peru.

Palavras-chave: Colômbia, Peru, cooperação internacional, desenvolvimento, fronteira, segurança. 
La frontera colombo-peruana tiene una extensión de 1.626 kilómetros (Cancillería de Colombia, 2021), a través de la cual Colombia y Perú mantienen relaciones diplomáticas desde el 16 de julio de 1822, siempre con el objetivo de estrechar y mantener los lazos históricos de amistad y cooperación, sobre la base del desarrollo de interacciones dentro del marco del diálogo directo, franco y cordial. A su vez, se han mantenido como principios fundamentales de la relación el respeto mutuo y la utilización de la diplomacia para la resolución de controversias, así como la no injerencia en los asuntos internos, para garantizar la estabilidad y la seguridad (Novak \& Namihas, 2011).

No obstante, la relación bilateral ha atravesado situaciones complejas, entre las que se puede mencionar la Guerra colombo-peruana, desarrollada entre septiembre de 1932 y mayo de 1933, catalogada como un conflicto limítrofe por la posesión de Leticia, ahora capital del departamento del Amazonas (Ávila Sánchez, 2017). Como antecedente en relación con la disposición limítrofe, es necesario mencionar el Tratado Lozano-Salomón, del 24 de marzo de 1922, que buscó acordar entre Colombia y Perú una frontera para delimitar, de una vez para siempre, el territorio de ambas naciones. Con el desarrollo del Tratado, se profundizó en el denominado Tratado de Navegación y Límites, en el que se establece que

\footnotetext{
Perú recibe los espacios comprendidos entre la margen derecha del Río Putumayo, hacia el oriente de la boca del Cuhimbé, y la línea establecida y amojonada como frontera entre Colombia y el Ecuador en las hoyas del Putumayo y del Napo. Por su parte Colombia recibe los territorios comprendidos entre el amojonamiento banda derecha del río Yaguas (desembocadura en el Putumayo), banda izquierda del río Atacuarí (desembocadura en el Amazonas) y de este punto a la frontera con el Brasil (Tabatinga-río Putumayo) (Ávila Sánchez, 2017, p. 159).
}

Con el incremento del conflicto fronterizo, la búsqueda de una salida diplomática se presentó desde el lado peruano con la solicitud de una Comisión Conciliadora; sin embargo, el Gobierno de Colombia, que se caracterizaba por su tendencia nacionalista, se negaba a finalizar la disputa mediante un arbitraje internacional, incentivando, en contravía diplomática, el recrudecimiento de las acciones militares. Para ello fue determinante el rápido desarrollo de la Fuerza Aérea Colombiana, que, a partir de su participación en este conflicto, estableció un hito en su configuración (Ávila Sánchez, 2017; Fuerza Aérea Colombiana, 2021).

Tras el fin de la guerra, las relaciones entre los dos países se afianzaron. El origen común por cuenta de la campaña libertadora de Simón Bolívar, las semejanzas culturales y sociales acercaron a Colombia y a Perú en torno a la búsqueda de un crecimiento económico para sus ciudadanos y la construcción de países industrializados. Por supuesto, la proximidad territorial coadyuvó a acercar a las poblaciones ubicadas en zonas de frontera y a que cooperaran para conseguir unas condiciones de seguridad local adecuadas (González-Cuenca \& Rodríguez, 2018), factores que fueron fundamentales en el establecimiento de escenarios políticos y comerciales más amplios, integradores y centralizados, como la Comunidad Andina (CAN), la Unión de Naciones Suramericanas (Unasur) y la asociación bilateral para negociar el Tratado de Libre Comercio (TLC) con la Unión Europea (Álvarez \& Guerra-Barón, 2013).

Aquí hay, entonces, una demostración de ambos Estados por mantener unas relaciones diplomáticas estables, determinadas por el vínculo territorial y las condiciones bilaterales que se han creado. La estrategia que estos países han consolidado con la puesta en marcha de la Operación de Apoyo al Desarrollo Binacional (OADB) es parte de ese proceso de relaciones bilaterales.

Pasando por el establecimiento de la Comisión de Vecindad e Integración Peruano-Colombiana (CVIPC), en el 1994, y la Comisión Binacional de Seguridad Fronteriza (Combifron), instalada en 2007, la OADB se presenta como un hito diplomático multidimensional de largo aliento, que busca seguir fortaleciendo y ampliando las áreas en las que pueden trabajar juntos, dentro del mantenimiento de una buena cooperación internacional que les permita reafirmar las acciones conjuntas para superar las dificultades de seguridad que vive la región compartida. 
Por lo anterior, este artículo se centrará en analizar las implicaciones, en materia de seguridad y desarrollo, de la implementación de la OADB en la frontera colombo-peruana, durante el periodo 2007-2019, basado en los conceptos de seguridad multidimensional y de desarrollo sostenible.

Es importante resaltar que la zona de frontera colombo-peruana presenta un problema de seguridad y desarrollo importante, producto de las organizaciones al margen de la ley y el narcotráfico, tradicionalmente originario del lado colombiano y que el escenario de posconflicto propuesto por la finalización del conflicto armado de Colombia con la guerrilla de las FARC solo recrudeció al desplazar la violencia y trasladar nuevos factores de conflicto hacia las zonas de frontera (González-Cuenca \& Rodríguez, 2018).

Es común destacar, también, la baja presencia estatal en los territorios más alejados del centro decisorio, tanto de Colombia como de Perú. No obstante, para el territorio estudiado, desde el lado colombiano, se ha producido, en los últimos 15 años, el auge de posiciones estatales de importancia relativa para garantizar mínimos en torno a la seguridad. Así, la Armada de Colombia, a través de la Fuerza Naval y la Marina de Guerra del Perú, ha mantenido la continuidad en el desarrollo de la Campaña Binacional Colombia-Perú desde el 2007 (Cancillería de Colombia, 2019).

\section{Método}

\section{Metodología aplicada}

Este artículo es un análisis cualitativo de corte analítico, que, según Roberto Hernández Sampieri (2014), "se fundamenta en una perspectiva interpretativa centrada en el entendimiento del significado de las acciones de seres vivos, sobre todo de los humanos y sus instituciones (busca interpretar lo que va captando activamente)" (p. 9). El estudio se ha fundamentado en la recolección de fuentes primarias, en las que, por medio de conversaciones extraoficiales de integrantes del equipo investigador, se pudo conocer la percepción de algunos habitantes de la región fronteriza y miembros de la Armada Nacional de Colombia que participaron en la Operación; y secundarias, integradas por información proveniente de las agencias, organismos e instituciones estatales de Colombia relacionadas con la OADB, como la Cancillería colombiana, la Armada Nacional de Colombia y el Departamento de Acción Integral de la Fuerza Naval del Sur, principalmente. También se basa en libros resultados de investigación y artículos académicos de bases virtuales de alto reconocimiento internacional, como Dialnet, Jstor, Latindex, Proquest, Scopus y Springer.

En algunos espacios, esta investigación se apoya en experiencias de los pobladores de la zona fronteriza que han sido beneficiarios de la OADB, las cuales han sido recolectadas por el equipo investigadores de manera informal, a partir de conversaciones, pero de las que se pudieron obtener percepciones generales sobre la Operación, dada la negativa de las personas a figurar por miedo a represalias de grupos ilegales que aún existen en la zona. También se ha reunido una serie de comentarios extraoficiales, brindados de manera espontánea por algunos miembros de las instituciones que participan en la Operación, como miembros activos de la Armada Nacional de Colombia, que sirven de apoyo a los análisis realizados. La información personal ha sido contrastada con los informes de instituciones oficiales, presentados por la Cancillería de Colombia y el Departamento de Acción Integral de la Fuerza Naval del Sur, para ser incluidos en el estudio y se ha desarrollado el tratamiento de la información personal de manera adecuada, bajo los parámetros éticos institucionales y nacionales.

\section{Fases metodológicas}

De manera paralela a la implementación de las nueve fases metodológicas que se describen más adelante, se desarrolló un marco teórico a partir del método de mapeo (Hernández Sampieri, 2014), para profundizar en la revisión de documentos teóricos relacionados con la seguridad multidimensional y el desarrollo sostenible. Con ello, se establecieron las principales definiciones, las dimensiones que integran los conceptos teóricos y las implicaciones analíticas que pueden 
desarrollarse en función del objeto de estudio propuesto, por lo que se descartaron aproximaciones relacionadas con la seguridad y el desarrollo que no abordaban aspectos prácticos o idóneos para el tratamiento de las condiciones resultantes de la implementación de la OADB. Tal es el caso de la seguridad vista de manera tradicional por el paradigma realista de las relaciones internacionales, o, en el caso del desarrollo, la concepción ligada al crecimiento económico. Como resultado, se consideraron la seguridad multidimensional y el desarrollo sostenible como enfoques teóricos para aplicar en el proceso investigativo y analítico.

De la recolección de información institucional, es importante destacar la aportada por la Armada Colombiana sobre el desarrollo de la OADB, desde el 2007 hasta el 2019, que se presenta como una fuente cualitativa y cuantitativa de alta importancia para el estudio, ya que identifica las acciones puestas en marcha de manera conjunta por los dos países, los resultados obtenidos de la operación y la evaluación del impacto de aquellas en función de las variables conceptuales de la seguridad multidimensional y el desarrollo sostenible en la población ribereña de los ríos Putumayo y Amazonas, tanto del lado peruano como colombiano.

Este artículo se enmarca en un proceso de investigación formativa denominado Proyectos de Iniciación Científica, que busca integrar de manera directa un investigador profesional con un estudiante para desarrollar un proyecto que busque respuestas a problemas nacionales e internacionales. Por lo cual, el proyecto presentó nueve fases metodológicas, de las cuales la primera era la definición de la idea específica que se requería trabajar, en donde la experiencia de los investigadores, desde el aspecto investigativo y aplicado, dio como resultado la determinación de una aproximación analítica de la OADB, que se presentó como un aspecto relevante desde la estructura de la fuerza naval de Colombia y de alta importancia para identificar los factores de cooperación en escenarios diplomáticos y militares entre los países fronterizos, para así mejorar las condiciones de las poblaciones que habitan en esos territorios.

Para el desarrollo de la segunda fase metodológica, los investigadores abordaron el planteamiento del problema, que se caracterizó por la necesidad de identificar los resultados que, en materia de seguridad multidimensional y de desarrollo sostenible, se han obtenido luego de 12 años de ejecución de la operación en territorio fronterizo, conduciendo así a la tercera parte del método, la cual consistió en la inmersión inicial en el campo, caracterizada por la búsqueda de información oficial relacionada con los objetivos, implementación y resultados obtenidos tras la operación año tras año.

Como cuarta fase, los investigadores elaboraron el diseño del estudio, que constaba, por un lado, de la presentación de aspectos históricos relacionados con las situaciones vividas entre Colombia y Perú para demostrar la evolución de estas, lo que permitió la consolidación de procesos de cooperación como la OADB; $y$, por otro, de la revelación de los resultados obtenidos en las estrategias más cercanas para validar un progreso en términos de seguridad multidimensional y desarrollo sostenible. A partir de ello, en la quinta fase se procedió a definir el periodo del estudio, el cual se determinó de acuerdo con los años de realización de la OADB, implicando, así, la recolección de datos específicos y su correspondiente análisis en la sexta y séptima etapa de la investigación, respectivamente.

Mediante una metodología de tipo cualitativa de corte analítico-descriptivo, se procesaron los resultados arrojados por la revisión de la información de fuentes académicas e institucionales, aplicada en todas las fases. Se desarrolló la octava fase, determinada por la interpretación de los resultados, lo que dio paso, como novena fase, a la conclusión del proceso metodológico diseñado, caracterizada por la elaboración del reporte de resultados, que se presenta en este artículo.

\section{Resultados}

\section{La operación de apoyo al desarrollo binacional Colombia-Perú}

La OADB surgió en el 2007 con el objetivo de incentivar las estructuras de las comunidades en relación con el desarrollo sostenible y el mejoramiento de las condiciones sociales multidimensionales de los habitantes 
de la zona fronteriza (Cancillería de Colombia, 2019), como una acción que se da entre las dos naciones y varias agencias estatales, que, mediante el fortalecimiento de la presencia del Estado, procuraba

[...] el mejoramiento de la calidad de vida de los pobladores en ambas riberas de los ríos Putumayo y Amazonas, que sirven de límite entre los dos países. Durante las jornadas, los pobladores recibieron atenciones en salud, servicios de medicina general y especializada, odontología, laboratorio clínico, valoración nutricional, cedulación, registro, charlas de prevención y promoción, así como la entrega en calidad de donación de paquetes alimentarios por parte del ICBF y elementos de aseo, ropa y vestuario (Cancillería de Colombia, 2019).

Durante los 13 años de la OADB, se han beneficiado 173.513 personas con 427.204 servicios de salud, según el informe presentado por la Armada de Colombia (2019). Este importante evento que busca reafirmar la soberanía nacional y estrechar lazos de hermandad entre ambos países, realiza su recorrido por los ríos Putumayo y Amazonas. Tripulantes de ambas fuerzas navales navegan más de 2.000 kilómetros, durante más de 90 días, llevando atención médica en diferentes especialidades, actividades lúdico-recreativas y más de 180 toneladas de ayudas humanitarias a los habitantes de este territorio fronterizo (Armada de Colombia, 2019).

Esta operación involucra a más de 80 hombres y mujeres de la Armada de Colombia y personal civil; de igual manera, la Marina de Guerra del Perú involucra a más de 70 personas, entre militares y civiles, que forman parte de estas travesías, durante las cuales han recorrido la ribera de los ríos Putumayo y Amazonas, llegando a más de 60 puntos, entre veredas y corregimientos, de diversos municipios de Colombia y Perú, en su primera edición en 2007; y de 117 -59 de esas poblaciones son colombianas y 58 , peruanas-, en sus últimas versiones, para beneficiar a más de 19.000 personas (Cancillería de Colombia, 2019).

Esta operación ha buscado integrar a más instituciones cada año, entre ellas la representación diplomática de Estados Unidos, particularmente apoyada por
Colombia, varios ministerios y agencias gubernamentales que atienden asuntos de salud, seguridad social y protección de infancia y desarrollo sostenible, al igual que instituciones de educación, como el SENA, lideradas por la Fuerza Naval del Sur, apuntando al establecimiento de acciones en pro de la paz, las relaciones internacionales y la consolidación de escenarios de seguridad. Con Perú, ocurre lo mismo con el liderazgo de la Marina de Guerra y organizaciones gubernamentales de apoyo multidimensional (Cancillería de Colombia, 2019).

Desde el 2007, el esfuerzo es mayor y con más instituciones involucradas en esta operación de gran magnitud, como el Ministerio de Agricultura y Desarrollo Rural, la Agencia Nacional para la Superación de la Pobreza Extrema (ANSPE), Artesanías de Colombia, Bancoldex, Invías, Migración Colombia y otras descritas en el Conpes 3.805 del 2014. Mediante el desarrollo de las campañas de sensibilización para contrarrestar los factores de inestabilidad en temas de seguridad multidimensional, se sigue buscando negarle el apoyo de los pobladores a las organizaciones al margen de la ley y al narcotráfico. Se ha procurado la mejora continua en cada versión, con lo cual, según el Departamento de Acción Integral de la Fuerza Naval del Sur, en el 2007, lograron un total de 10.000 servicios prestados y 4.794 personas beneficiadas, y, terminado el 2019, con 24.600 servicios prestados y 10.561 personas beneficiadas, lo que incrementa así, para cada edición, los servicios prestados y la cantidad de personas atendidas.

Tabla 1

Resultados obtenidos por la OADB del 2007 al 2019

\begin{tabular}{c|c|c}
\hline Año & Total servicios prestados & Total personas beneficiadas \\
\hline 2007 & 10.000 & 4.794 \\
\hline 2008 & 22.215 & 10.405 \\
\hline 2009 & 27.595 & 16.000 \\
\hline 2010 & 26.484 & 12.890 \\
\hline 2011 & 26.767 & 9.579 \\
\hline 2012 & 25.175 & 9.816 \\
\hline 2013 & 27.989 & 11.726 \\
\hline 2014 & 26.989 & 16.016 \\
\hline 2015 & 45.346 & 19.135 \\
\hline 2016 & 53.188 & 19.156 \\
\hline 2017 & 52.236 & 19.032 \\
\hline 2018 & 58.621 & 14.403 \\
\hline 2019 & 24.600 & 14.403 \\
\hline Total & 427.204 & 173.513 \\
\hline
\end{tabular}

Fuente: Departamento de Acción Integral Fuerza Naval del Sur (2019) 
Mediante las estrategias establecidas por la Operación para identificar directamente las condiciones de la población se han impulsado de manera sostenible los proyectos productivos que sean acordes a la región, para que los pobladores puedan ser autosuficientes y manejar dichos proyectos de manera autónoma, valor que permitirá continuidad en los proyectos y la sostenibilidad en función del desarrollo de la región, evitando así que recaigan en procesos ilícitos.

\section{Seguridad multidimensional y desarrollo sostenible a partir de la OADB}

La OADB tiene el compromiso de trabajar en campañas productivas e impulsar el desarrollo sostenible para garantizar resultados en los ámbitos económico, ambiental y social como beneficio para las comunidades. Con el progreso en la frontera colombo-peruana se garantizará una vida sana para los pobladores, la educación inclusiva como aporte a la generación de trabajo en la frontera, con lo cual se promueve la colaboración de nuevas instituciones que se han ido involucrando a lo largo de las trece versiones de la Operación.

Para la representación de la Organización de las Naciones Unidas (ONU) en el territorio, es necesario que los estamentos gubernamentales puedan fomentar alternativas de mejoramiento compartidas con las comunidades indígenas. Las acciones aisladas y parcializadas no pueden ser una constante y la desarticulación interinstitucional y social se presenta como una debilidad en la estrategia que se ha presentado, afectando el uso de los recursos destinados para la atención de los problemas. Según la onu (2020, p. 6), los canales de comunicación deben ser mejorados, pues son la causa fundamental de la desarticulación mencionada. Se hace imperativo establecer, entonces, una comunicación efectiva para llevar lo que producen estas comunidades a los mercados del país.

Además, la OADB se ha enfocado en actividades relativas a la paz y las relaciones internacionales, así como a la defensa y la seguridad de las naciones en áreas de frontera, por lo cual lo más satisfactorio ha sido escuchar de parte de los mismos pobladores, según relatos de los integrantes de la Operación, que su calidad de vida les está cambiando, que se sienten más seguros, que los proyectos productivos que los gobiernos han impulsado en sus territorios les están dando un gran impulso para progresar económicamente y desarrollarse de manera sostenible. Los pobladores dicen sentirse felices de estar del lado de la legalidad, con proyectos inclusivos y que le están aportando al crecimiento de la región y a la eliminación de estigmas. Pero quizá la satisfacción más grande de las personas es reconocer que se volvió un hábito positivo el que, año tras año, el Estado llegue a su territorio con las instituciones necesarias para ayudar en su crecimiento personal, familiar y comunitario, como una atención más fuerte del ICBF, del Ministerio de Educación Nacional, del Ministerio de Ambiente y Desarrollo Sostenible, por mencionar algunas, y así poder acceder a programas de los que no habían escuchado antes. Esto les muestra una posibilidad de construir un nuevo futuro.

De las 13 versiones de la OADB, se ha identificado que la decisión de los gobiernos de ambos países ha sido la de aumentar su presencia estatal en esta región tan alejada de las ciudades capitales y, a la vez, fomentar la vinculación institucional, tanto pública como privada, en esta gran labor, con el propósito de lograr articular proyectos sostenibles que permitan el fortalecimiento social y les brinden a las comunidades un crecimiento económico que expulse de sus territorios los cultivos ilícitos y disminuya el atractivo de participar en el narcotráfico, lo cual conduce a mejorar la seguridad.

La percepción del avance en materia de seguridad y desarrollo, desde la perspectiva de los pobladores de la región, es bastante alentadora. Según la poca información suministrada por los mismos pobladores a los integrantes de la Operación, aquello que identifican como el factor más difícil de asumir en las poblaciones que han estado bajo la presión y zozobra de las amenazas de los grupos al margen de la ley y del narcotráfico es tener contacto con personal de las instituciones del Estado y la Fuerza Pública por miedo a las represalias que puedan tomar estas organizaciones criminales, por lo cual, en un alto porcentaje, deciden guardar silencio y, en ocasiones, no recibir ninguna 
ayuda proveniente de los estados. Sin embargo, gracias a la periodicidad de la OADB, que les ha ido brindando mayor respaldo, alineada con la misión de la Armada Nacional de Colombia, la Fuerza Naval del Sur, con su puesto de mando en Puerto Leguízamo, Putumayo, quienes han enfocado sus esfuerzos para prestar sus servicios, entre los cuales se destaca la actividad de seguridad en los ríos Putumayo, Caquetá y Amazonas, han podido construir escenarios de confianza recíproca, ofreciéndole tranquilidad a los habitantes de esta región del país.

La recurrencia de la OADB se muestra como una de sus grandes virtudes. Debido a esto, se ha logrado transformar la percepción de seguridad y desarrollo de los pobladores de la zona ribereña de los ríos Putumayo y Amazonas y ha aumentado la presencia estatal, principalmente en programas como la sustitución de cultivos ilícitos, con lo cual los pobladores han encontrado una nueva oportunidad laboral, produciéndose un incremento en la motivación para seguir trabajando y para erradicar totalmente de sus territorios estos cultivos.

A pesar de esto, los mismos pobladores, de manera extraoficial, reconocen que falta mucho trabajo, $y$, más aún, por parte del Estado colombiano, en lo referente a las vías de comunicación, terrestres y fluviales, dado que les es muy poco rentable cultivar productos que deben ser comercializados en mercados de municipios fronterizos del departamento de Putumayo y ciudades como Leticia, en el departamento de Amazonas, desde donde son transportados al resto del país y fuera de este, ocasionando sobrecostos por los precios de los fletes y la intermediación. En contraparte, los pobladores perciben el cultivo y venta de la hoja de coca como una opción importante, ya que las organizaciones ilegales se las compran directamente en sus casas, y pagan muy bien el producto.

Sin embargo, los pobladores reconocen que el terreno que la Armada de Colombia y la Marina de Guerra del Perú le han negado a los grupos al margen de la ley y al narcotráfico les produce tranquilidad y seguridad, así como la posibilidad de ver crecer a sus hijos felices a su lado, sin ser arrebatados por los grupos armados, a causa del reclutamiento forzado, que ha sido considerado como un factor de alta importancia en estos territorios.

En términos generales, los pobladores de la región fronteriza han encontrado en la OADB la oportunidad, a partir de la llegada del Estado, para potencializar el desarrollo de sus territorios, lo cual les ha traído mejor calidad de vida y progreso en varias dimensiones de la seguridad. La Operación se convirtió en el primer peldaño para recuperar el territorio históricamente dominado por los grupos al margen de la ley y el narcotráfico, siendo, a la vez, impulsora de los proyectos productivos que deben conducir a la consolidación de la soberanía por cuenta de la institucionalidad estatal. De esta manera, se ha convertido en el programa de cooperación e integración más importante en términos de seguridad y desarrollo sostenible que se ha desarrollado entre Colombia y Perú, así como el que más y mejores resultados, en términos multidimensionales, ha arrojado en beneficio de la población fronteriza de ambos países.

\section{Discusión}

\section{Seguridad multidimensional en la frontera colombo-peruana}

La frontera compartida entre Colombia y Perú se ha caracterizado por momentos de incertidumbre, como la guerra librada a inicios del siglo xx por cuestiones territoriales sobre Leticia. Las condiciones geográficas y la falta de comunicación tradicional con las ciudades capitales se suman al recuerdo de la guerra vivida. La imagen de ser retaguardia de grupos armados ilegales de Colombia y un territorio geoestratégico para el narcotráfico es permanente, no solo en los habitantes de la zona, sino en todos los ciudadanos de ambos países (López \& Tuesta, 2015).

Lo anterior puede ser corolario de las dificultades estructurales que afrontan las poblaciones de estos territorios, que se conforman en su mayoría por comunidades indígenas. La denominada Triple Frontera Amazónica, configurada por territorios colindantes 
de Brasil, Colombia y Perú, es un territorio donde se ubican más de 400 comunidades indígenas, albergando, según cifras de la onU (2020), cerca de 6 millones de personas. El territorio de la Triple Frontera que incluye territorio brasileño abarca una población de 208.699 personas, de la cual el $57 \%$ es caracterizado como indígena.

En Colombia, en el Amazonas, las cifras de inseguridad alimentaria presentadas por la onu (2020) dan cuenta de un $59 \%$ de la población en condición moderada o grave, mientras que en Perú se informa de un $49 \%$ de la población en estas mismas condiciones. Es claro que el fenómeno de desabastecimiento y parálisis de los medios de transporte fluviales y aéreos provocado por la pandemia por la covid-19 ha influido en el incremento de las cifras; sin embargo, la condición en estas poblaciones se asume de manera estructural, pues el mismo informe presenta un desabastecimiento previo a la pandemia de un $28 \%$, recalcando, también, la desnutrición infantil, toda vez que casi la mitad sufren de anemia.

Algo similar sucede con la prestación de servicios de salud: el acceso es de baja calidad y la cantidad de lugares es insuficiente para atender condiciones de emergencia; tanto equipos como personal e insumos se han vuelto escasos y de difícil consecución (ONU, 2020 , p. 5). Es decir, el problema del territorio fronterizo se presenta de manera multidimensional, impactando contundentemente el factor de seguridad, incluyendo condiciones ligadas a la salubridad y al agua potable, así como los elementos dispuestos para la higiene; no hay garantías para las familias de poder suplir las necesidades alimentarias básicas, a causa de una baja en los ingresos y las dificultades para acceder a productos comercializables.

Asimismo, las variables relacionadas con la seguridad multidimensional (la violencia de género, la trata de personas, el trabajo infantil, la deforestación por el comercio de madera y la expansión de la frontera agrícola) han sido impactadas negativamente de manera constante (Equipo de Modelación y Niveles de Referencia Sistema de Monitoreo de Bosques y Carbono, 2018).

En el aspecto tradicional de la seguridad, se pueden destacar el narcotráfico, que, a partir de la movilización poco regulada que existe por los ríos Amazonas y Putumayo, puede transportar narcóticos hacia ambos lados de la frontera, para ser enviados a Norteamérica, Europa y Asia, principalmente (García Pinzón, 2018). Además, es necesario vincular el desarrollo acelerado que han tenido en este territorio las organizaciones de crimen organizado trasnacional, que representan una nueva amenaza para la seguridad de ambos países (Trejos \& Puente, 2016).

\section{Desarrollo sostenible en la frontera colombo-peruana}

El desarrollo sostenible debe darse de manera que sea equitativo, debe buscar el bienestar de las personas como principal prioridad, por lo cual la OADB encaja a la perfección con estas pretensiones que, desde el Gobierno nacional, se articulan a la agenda emanada de los Objetivos de Desarrollo Sostenible, como apoyo al desarrollo en esta zona.

Para el caso fronterizo colombo-peruano, se entiende que la gestión ambiental es una herramienta clave para avanzar en materia de desarrollo. La biodiversidad alojada en esta área, los bienes hídricos y la caracterización de la Amazonía en su conjunto como un bien supremo de la humanidad dejan ver que, para las dos naciones, el desarrollo sostenible debe ser considerado un pilar dentro de cualquier marco de entendimiento binacional o plan de acción unilateral.

En este sentido, las comunidades indígenas, por su cosmovisión ligada con la protección ambiental y el aprovechamiento sostenible de los bienes naturales, pueden aportar un valor agregado, en función del desarrollo sostenible y la interacción de las poblaciones fronterizas. En perjuicio de lo anterior, Ladino y Rey (2010) concluyen que:

[...] la gestión ambiental ocurre solo en el marco de cada municipio fronterizo, pero no es un tema que haya unido a los actores y entidades del ámbito fronterizo, además persisten problemas como contaminación de aguas, basuras, destrucción de recursos naturales, tráfico de recursos naturales, falta de educación ambiental, 
entre otros, a pesar del esfuerzo realizado por las entidades ambientales de Leticia (p.47).

En consonancia con el sentido del desarrollo sostenible de la frontera, se puede destacar el Proyecto Putumayo Tres Fronteras - Colombia, Ecuador y Perú, financiado, desde el 2011, por la Unión Europea y la Organización WWF Alemania, que tiene por objetivo:

[...] contribuir a la conservación de la biodiversidad y al desarrollo sostenible de la cuenca media del río Putumayo en el marco de los acuerdos y compromisos ambientales multilaterales adquiridos por Colombia, Ecuador y Perú... tener incidencia en políticas ambientales, públicas y sectoriales de ámbito nacional y regional, mediante el desarrollo herramientas y mecanismos operativos, técnicos y financieros (WWF, 2011).

\section{Relaciones bilaterales con enfoque fronterizo entre Colombia y Perú}

Los procesos de integración y las acciones conjuntas entre los Gobiernos de Colombia y de Perú se han desarrollado desde la misma fundación de la CAN en 1969; no obstante, los enfoques en esta zona fronteriza se dan tan solo hasta 1988, cuando, a partir del funcionamiento de la Comisión Mixta del Tratado de Cooperación Amazónica Peruano-Colombiano, se inicia un proceso que redundaría en la configuración del Plan Peruano-Colombiano para el Desarrollo Integral de la Cuenca del Río Putumayo, aprobado diez años más tarde, demostrando las complicaciones diplomáticas para concretar acciones reales para la zona de frontera (Novak \& Namihas, 2011).

Dado el desarrollo de las relaciones en el interior de la CAN, en 1999 se presenta el Plan de Desarrollo de la Zona de Integración Fronteriza (ZIF), que formó parte de la Política de Integración y Desarrollo Fronterizo de la organización multilateral, con el respaldo de la antigua CAF, hoy Banco de Desarrollo de América Latina y el Banco Interamericano de Desarrollo (BID) (Novak \& Namihas, 2011).
El Plan cuenta con siete objetivos estratégicos, líneas de acción y sus correspondientes metas e indicadores, debidamente formulados; resultado de investigaciones en los propios territorios y en consulta con las comunidades locales. Como resultado el Plan de Desarrollo está conformado por seis grandes Programas que coinciden con los Objetivos Estratégicos planteados en el marco del Plan de Desarrollo de la zIF peruano colombiana. Dentro de los proyectos prioritarios se incluyen también intervenciones encaminadas al suministro de energía proveniente de fuentes alternativas, manejo de bosques, y aspectos relacionados con la logística de comercialización o intercambio de bienes utilizando el transporte fluvial; los cuales están asociados también a las necesidades más apremiantes de las poblaciones que integran los [Núcleos de Desarrollo e Integración] NDIs de las [Unidades Geoeconómicas] UGE del Putumayo. El trabajo así mismo incluye los mecanismos de gestión del Plan, la Institucionalidad Política y Operativa, y la propuesta de la creación de un Fondo Binacional para el Desarrollo de la ZIF con aportes provenientes de los gobiernos de Perú y Colombia, de instituciones multilaterales, países pertenecientes a la OCDE y otros. Además propone los mecanismos de Gestión del Plan desde la visión institucional, política y operativa y propone una Comisión Binacional Autónoma de Gestión de la zIF y reglamentos en diferentes materias, como el sistema de monitoreo del Plan (sistematización, seguimiento y evaluación). Es de destacar que si bien el territorio de la ZIF es demasiado grande y poco poblado, dada su homogeneidad en las características físicas-ambientales y económico-sociales, ha sido acertado trabajar en su totalidad la región para la elaboración y ejecución del Plan (Arciniegas, 2018, p. 31).

Más adelante, a inicios de los 2000, se da origen a la Comisión de Transición del Plan Peruano-Colombiano para el Desarrollo Integral de la Cuenca del río Putumayo, con un componente sostenible hacia el manejo y la gestión de los bosques de la Amazonía, incluyendo una visión de comercialización de productos nativos, con la administración adecuada de los recursos naturales, teniendo presente factores como el cambio climático, considerado ya como una amenaza 
para la seguridad, y los aspectos relacionados con el uso de maderas y productos artesanales. Así se vinculan los valores de la seguridad multidimensional y el desarrollo sostenible por primera vez en los planes de cooperación e integración binacional (Novak \& Namihas, 2011).

Para realizar seguimientos, actualizaciones y modificaciones previas a este plan, en el 2003, se creó el Comité Técnico Binacional de Desarrollo e Integración Fronteriza de la Comisión de Vecindad e Integración Peruano-Colombiana, que funcionó hasta 2010, cuando el plan ya estaba en ejecución (Novak \& Namihas, 2011).

En concordancia con el proceso regional de integración y cooperación, desde el 2007, se desarrollaron negociaciones por cuenta de Perú y Colombia, en el marco de la CAN, para acordar un Tratado de Libre Comercio con la Unión Europea, en el entendido de la negociación entre bloques. Los acercamientos de alto nivel entre los países andinos no solo dieron un nuevo aire a la organización multilateral, que, luego, por dificultades con Bolivia y Venezuela, se complicaron, sino que afianzó las relaciones bilaterales, promoviendo un objetivo común. Finalizó en el 2010 con el hecho de que los líderes de ambas partes firmaron las bases comerciales del acuerdo (Álvarez \& Guerra-Barón, 2013, p. 128).

Todos estos esfuerzos por propiciar acercamientos bilaterales, procesos de integración y puntos de acuerdo demuestran que, para el 2007, el lanzamiento de la OABD se configura como la punta de lanza de un proyecto direccionado a mejorar radicalmente las condiciones de seguridad multidimensional de la zona fronteriza de Colombia y Perú, a la par que se configuran y consolidan escenarios de desarrollo sostenible para la población ribereña de los ríos Amazonas y Putumayo.

Para el 2011, los presidentes de ambos países acordaron instaurar el Mecanismo de Consulta y Coordinación $(\mathrm{MCC} 2+2)$-la instancia más alta para definir aspectos relacionados con la lucha conjunta contra el terrorismo y el narcotráfico, con un énfasis específico en la zona fronteriza-, estableciendo que la seguridad es una prioridad y la base del desarrollo integral de este territorio (Cancillería de Colombia, 2021).
En el mismo sentido, con el MCC2+2 se presenta una importante relación entre la seguridad multidimensional y el desarrollo sostenible, por ejemplo, al

[...] coordinar entre las Fuerzas Armadas y la Policía Nacional de ambos países, y a través de ellas con otras entidades nacionales, el diseño y ejecución de acciones para preservar y proteger el medio ambiente y la biodiversidad amazónica, ante las diferentes amenazas y riesgos de seguridad: a) Minería ilegal. Desarrollar operaciones conjuntas, coordinadas y sincronizadas con el fin de identificar, combatir y erradicar la explotación ilegal, especialmente, en los dos Putumayo, Amazonas y sus afluentes. b) Explotación y comercio ilegal de maderas. Coordinar acciones e intercambiar información para proteger los recursos maderables de la Amazonía y desarrollar actividades de interdicción, especialmente, sobre los dos Putumayo, Amazonas y sus afluentes. c) Tráfico de especies de fauna y flora silvestre. En el marco de su competencia, adoptar medidas para proteger y preservar las especies de fauna y flora silvestre, y combatir su tráfico. d) Depredación de la selva amazónica. Fortalecer las capacidades de prevención y respuesta, en el ámbito de sus competencias, contra las actividades de depredación de la selva amazónica (Cancillería de Colombia, 2021).

Este mecanismo se ha establecido dentro de la Combifron, que integra reuniones de alto nivel de las instituciones militares, las organizaciones regionales que tienen lugar en la zona de frontera y los niveles relacionados con seguridad y defensa nacional de ambos países, y así también se incluye el Plan Operativo Anual Binacional (РОAB) (Cancillería de Colombia, 2021).

Uno de los grandes valores para que la Operación sea una realidad ha sido la configuración de la zIF, que se enfoca principalmente en atender áreas económica y socialmente deprimidas y articularlas con zonas de alto potencial de recursos. La ZIF Colombia-Perú ha impulsado estrategias que buscan sacar este territorio del sometimiento y aprovechamiento por parte de los grupos al margen de la ley y el narcotráfico (Moreno Urigüen, 2006); la OADB ha estado trabajando para 
impulsar el desarrollo, llevar las instituciones del Estado a estas zonas alejadas y crear un ambiente de seguridad en la zona fronteriza.

También, entre la ZIF y la OADB existe unan interacción para asesorar e impulsar proyectos productivos que sustituyan los cultivos ilícitos, con el propósito de promover oportunidades para miles de colombianos que soportaron más de medio siglo de conflicto armado en lugares recónditos del país y aportar al desarrollo del lado peruano. Sin embargo, han encontrado grandes dificultades para la implementación de estos proyectos, debido a la mala infraestructura vial y el pésimo transporte fluvial en los ríos Putumayo y Amazonas.

Así, para el 2007, se desarrolló la Campaña de Apoyo al Desarrollo Colombia-Perú en el marco de la OADB, que:

[...] se trata de un esfuerzo binacional e interinstitucional, que tiene como objeto fortalecer la presencia Estatal y propender por el mejoramiento de la calidad de vida de los pobladores en ambas riberas de los ríos $\mathrm{Pu}$ tumayo y Amazonas, que sirven de límite entre los dos países (Cancillería de Colombia, 2019).

No obstante el impacto de la OADB, la pandemia por la covID-19 ocasionó una transformación radical en los esfuerzos presentes hasta el año de su aparición. Para la región, el contexto fue definido como crítico, lo que condujo a que las autoridades locales, nacionales e internacionales diseñaran una estrategia multidimensional con acciones necesarias para la región (onu, 2020, p. 7).

Para el desarrollo de este plan, Colombia y Perú, junto con Brasil, han logrado construir estrategias de cooperación que tienen una destinación de recursos cercana a los 10.500 .000 USD, de los cuales tan solo 1.500.000 USD se destinan a Brasil, mostrando el panorama de la necesidad de los otros dos países:

Como primer paso para poner en marcha el Plan, desde la oficina de OCHA Colombia se ha contratado un coordinador de terreno/analista de información en Leticia, con el fin de apoyar los datos, demás tareas de información y coordinar las evaluaciones y respuesta con los socios humanitarios e instituciones locales. Desde Perú se están buscando recursos adicionales para la contratación de dos personas que apoyen tareas similares en su territorio. Brasil está adelantando planes similares para contratar a una persona que esté basada en Manaos para apoyar la extensión de actividades a la triple frontera. El Plan de Acción cuenta inicialmente con un énfasis en la respuesta en los sectores de Salud, Seguridad Alimentaria y Nutrición (SAN) y Agua y Saneamiento Básico (WASH), identificados como prioritarios por la misión de evaluación y respondiendo también a las necesidades prioritarias identificadas por los Gobiernos. Las actividades de este plan de acción se resumen en 25 actividades críticas para implementación. La mayoría de estas actividades son comunes a las intervenciones requeridas en los tres países (ONU, 2020, p. 7).

En consecuencia, las relaciones bilaterales llegan a abordar incluso situaciones de amenaza imprevistas como la pandemia mundial, promoviendo la interacción bilateral entre Colombia y Perú, con injerencia de Brasil, por la Triple Frontera Amazónica, y la comunidad internacional, a través de organizaciones multilaterales.

\section{Conclusiones}

Si bien es cierto que las relaciones entre Colombia y Perú, a inicios del siglo xx, fueron, de una u otra manera, consideradas como tormentosas, debido a la guerra fronteriza y la delimitación de las fronteras a través del Tratado Lozano-Salomón, fueron siempre un soporte para los acercamientos diplomáticos y la resolución de conflictos consensuada.

Desde el desarrollo de la guerra en los años 30, las relaciones bilaterales se fortalecieron de una manera exponencial. Las diferentes acciones binacionales que se crearon y las interacciones gestadas en el interior de organizaciones multilaterales dan cuenta de la solidez de las relaciones y también de los esfuerzos, en 
diferentes niveles de la toma de decisión estatal, que han hecho ambos países por garantizar los mínimos vitales a la población habitante de la zona fronteriza.

Los ya mencionados comités, comisiones, planes, tratados, políticas binacionales y el Tratado de Libre Comercio negociado en el marco de la CAN con la Unión Europea, el MMC2+2 y el POAB, son la demostración del empeño por consolidar espacios de seguridad y desarrollo en la zona de frontera.

A partir de lo anterior, es posible dimensionar la importancia, el desarrollo y los resultados que, en materia de cooperación e integración, puede tener la constante implementación de la OADB Colombia-Perú, toda vez que ayuda, de manera decisiva, en la reducción de factores de inseguridad multidimensionalidad y apoya el desarrollo sostenible de la región. Ambos factores están intrínsecamente relacionados, pues el medioambiente, la seguridad ciudadana, el acceso a bienes naturales y su protección, la mejora en las condiciones de salud, alimento, vivienda, transporte, etc., son vitales para la región y se enmarcan como los objetivos de la Operación.

El acceso a las instituciones, el acompañamiento de estas y el fomento de actividades productivas de orden sostenible son pilares para consolidar esos escenarios de seguridad y desarrollo que promulga la OADB, a la vez que son factores favorables para la consolidación de escenarios de soberanía territorial, confianza en las instituciones estatales y el enfrentamiento estructural de amenazas como el terrorismo, el crimen organizado transnacional, el narcotráfico, la deforestación y el cambio climático.

La OADB, que, de 2007 a 2019, ha cumplido con cerca de 200.000 ciudadanos de ambos países, es recibida con los brazos abiertos y esperada anualmente con ansias. Las condiciones sociales de la región han cambiado y, poco a poco, los pobladores empiezan a transformar sus modelos productivos ilegales hacia la legalidad, restando espacio a los criminales; y, aunque aún persiste un grado importante de temor frente a las represalias que esto pueda provocar, las personas se vuelven más conscientes de su papel comunitario $\mathrm{y}$, en consenso con las comunidades indígenas, se ha empezado a notar un cambio importante en el pensamiento de la población, que permite concluir con un valor agregado de escenarios de optimismo en la zona fronteriza entre Colombia y Perú, gracias al desarrollo anual de la OADB Colombia-Perú.

Declaración de conflicto de interés: Los autores no manifiestan conflictos de interés institucionales ni personales.

\section{Referencias}

Álvarez, J. M., \& Guerra-Barón, A. (2013). Acuerdo de Asociación Colombia y Perú-Unión Europea: de negociación bloquea bloque a negociación multi-partes. Diálogos de Saberes, (38), 117-132. https://revistas.unilibre.edu.co/ index.php/dialogos/article/view/1824/1358

Arciniegas, H. (2018). La integración y el desarrollo fronterizo en la Comunidad Andina. Reflexiones sobre su origen y evolución. Aldea Mundo, (45), 23-35. https://www.re dalyc.org/jatsRepo/543/54356888003/html/index.html

Armada de Colombia (2019, 22 de julio). Culminó con éxito la actividad de bienestar más importante de la Amazonía colombiana. Armada de Colombia. https://www. armada.mil.co/es/content/culmino-con-exito-activi dad-bienestar-mas-importante-amazonia-colombiana

Ávila Sánchez, V. C. (2017, enero-junio). La guerra entre Colombia y Perú (1932-1934). Una perspectiva desde la prensa venezolana. Tiempo y Espacio, 35(67), 151-175. https:// dialnet.unirioja.es/servlet/articulo?codigo $=6175402$

Cancillería de Colombia (2019, 17 de mayo). Inició la jornada binacional Colombia-Perú. Cancillería de Colombia. https://www.cancilleria.gov.co/newsroom/news/ inicio-jornada-binacional-colombia-peru

Cancillería de Colombia (2021a). Plan de Acción para la seguridad fronteriza entre el Ministerio de Defensa Nacional de Colombia y los Ministerios de Defensa y del Interior del Perú. Bogotá: Ministerio de Relaciones Exteriores de Colombia. https://www.cancilleria.gov.co/sites/default/files/ Normograma/docs/pdf/plan_seguridadfronteriza.pdf Cancillería de Colombia (2021b, 19 de marzo). Frontera Terrestre Colombia-Perú. Cancillería de Colombia. https: //www.cancilleria.gov.co/frontera-terrestre-colombia-peru Cancillería de Colombia (2021c, 22 de marzo). Seguridad y Defensa. Cancillería de Colombia. https://www.cancilleria. gov.co/en/integracion-fronteriza-brasil-ecuador-y-peru 
Departamento de Acción Integral Fuerza Naval del Sur (2019). Estadísticas generales Operación Binacional Colombia-Perú, 2007-2019. https://www.armada.mil.co/sites/ default/files/informe_de_gestion_arc_-_2019.pdf

Equipo de Modelación y Niveles de Referencia Sistema de Monitoreo de Bosques y Carbono. (2018). Caracterización de las principales causas y agentes de la deforestación a nivel nacional 2005-2015. FAO, MASD, \& IDEAM. http://docu mentacion.ideam.gov.co/openbiblio/bvirtual/023780/ Caracterizacion.pdf

Fuerza Aérea Colombiana. (2021, 19 de marzo). El conflicto fronterizo. Fuerza Aérea Colombiana. https://www.fac. mil.co/v-el-conflicto-fronterizo

García Pinzón, V. (2018). Dimensiones locales de la seguridad y la cooperación transfronteriza en la frontera amazónica de Brasil, Colombia y Perú. OPERA, (23), 59-80. https:// doi.org/10.18601/16578651.n23.05

González-Cuenca, D., \& Rodríguez, E. (2018). Desarrollo de potencialidades fronterizas de Colombia en el posconflicto: una oportunidad para la gobernanza de la región Andina. En D. Calderón, \& D. Palma, Gobernanza Multidimensional (pp. 167-186). Ediciones USTA.

Hernández Sampieri, R. (2014). Metodología de Investigación (6. ${ }^{\mathrm{a}}$ ed.). McGraw Hill Education.

Ladino, W., \& Rey, O. (2010). Gestión Ambiental y Fronteras. Análisis de las dinámicas de los actores locales en el departamento del Amazonas. Nova et Vetera, (19), 33-48.

Leal, K. (2019, 11 de septiembre). Elecciones 2019: Violencia va en aumento, dice Fundación Paz y Reconciliación. RCN Radio. https://www.rcnradio.com/colombia/ elecciones-2019-violencia-va-en-aumento-dice-funda cion-paz-y-reconciliacion
López, N., \& Tuesta, D. (2015). Economías ilícitas y orden social: la frontera de Perú, Brasil y Colombia. CIDOB d'Aferes Internacionals, (111), 79-104. https://www.cidob.org/es/ articulos/revista_cidob_d_afers_internacionals/111/ economias_ilicitas_y_orden_social_la_frontera_de_pe ru_brasil_y_colombia

Moreno Urigüen, H. (2006). Las implicaciones del conflicto armado interno colombiano para las fronteras de Ecuador, Perú, Brasil y Venezuela, 2000-2005. Íconos, (24), 161-170. https://revistas.flacsoandes.edu.ec/iconos/ article/view/155/151

Novak, F., \& Namihas, S. (2011). Las relaciones entre Perú y Colombia. Perú-Colombia: La construcción de una asociación estratégica y un desarrollo fronterizo. Konrad Adenauer Stiftung. http://repositorio.pucp.edu.pe/ index/bitstream/handle/123456789/39908/Per\%c3\% ba-Colombia_La_construcci\%c3\%b3n_de_una_asocia ci\%c3\%b3n_estrat\%c3\%a9gica_y_un_desarrollo_fron terizo.pdf?sequence=1\&isAllowed=y

Organización de las Naciones Unidas (onu). (2020). Plan de Acción Triple Frontera Amazónica. Colombia-Brasil-Perú. Humanitarian Response. https://www.humanitarianres ponse.info/sites/www.humanitarianresponse.info/files/ documents/files/plan_de_accion_triple_frontera_vf.pdf

Trejos, L., \& Puente, M. de la (2016). El lado colombiano de la frontera con Perú. ¿Vacío, inseguro y poco integrado? Justicia, (30), 52-69. http://dx.doi.org/10.17081/just. 21.30.1349

WWF. (2011, 7 de febrero). Proyecto Putumayo Tres Fronteras-Colombia, Ecuadory Perú. https://wwfint.awsassets. panda.org/downloads/plegable_pt_completo_final_ 2010_1.pdf 